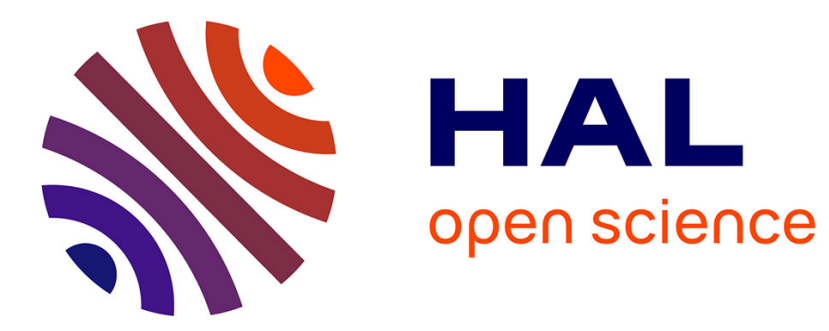

\title{
L'Ethnomusicologie de l'Amazonie
}

Jean-Michel Beaudet

\section{To cite this version:}

Jean-Michel Beaudet. L'Ethnomusicologie de l'Amazonie. L'Homme - Revue française d'anthropologie, 1993, 33 (126), pp.527-533. 10.3406/hom.1993.369655 . hal-01657396

\section{HAL Id: hal-01657396 https://hal.parisnanterre.fr/hal-01657396}

Submitted on 6 Dec 2017

HAL is a multi-disciplinary open access archive for the deposit and dissemination of scientific research documents, whether they are published or not. The documents may come from teaching and research institutions in France or abroad, or from public or private research centers.
L'archive ouverte pluridisciplinaire HAL, est destinée au dépôt et à la diffusion de documents scientifiques de niveau recherche, publiés ou non, émanant des établissements d'enseignement et de recherche français ou étrangers, des laboratoires publics ou privés. 


\section{L'Ethnomusicologie de l'Amazonie}

Jean-Michel Beaudet

\section{Citer ce document / Cite this document :}

Beaudet Jean-Michel. L'Ethnomusicologie de l'Amazonie. In: L'Homme, 1993, tome 33 n¹26-128. La remontée de l'Amazone. pp. 527-533;

doi : 10.3406/hom.1993.369655

http://www.persee.fr/doc/hom_0439-4216_1993_num_33_126_369655

Document généré le 29/03/2016 


\section{L'Ethnomusicologie de l'Amazonie}

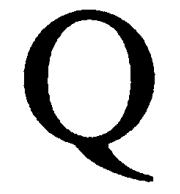

ue savons-nous des musiques amazoniennes ? Existe-t-il des caractéristiques générales communes aux musiques des basses terres d'Amérique du Sud ? Quels sont les traits distinctifs de ces musiques ? Peut-on parler d'une rythmique gé, d'une physionomie tonale karib, de formes mélodiques tupi ? Quelle est ici la pertinence d'une mise en relation des styles musicaux et des familles linguistiques ? On peut certes le regretter, mais il est encore impossible de répondre à ces questions ; aussi les ethnomusicologues amazonistes ne peuvent-ils se montrer que très méfiants à l'égard de toute généralisation touchant cette région. Les ouvrages substantiels sur le sujet se comptent sur les doigts d'une main ; l'ethnomusicologie de l'Amazonie n'a pas encore de profondeur historique. Nous ne disposons pas de l'équivalent des monographies des d'Harcourt $(1925,1959)$ sur les Andes. Nous avons bien les gravures et les narrations de Staden, Thevet et Léry au Xvi ${ }^{\mathrm{e}}$ siècle, puis les descriptions des siècles suivants, d'ailleurs souvent plus fragmentaires et marginales quant à la musique que celles des premières chroniques. Camêu (1977) offre, pour le Brésil, un panorama satisfaisant de ces références anciennes ; en revanche, son étude des instruments de musique apporte peu par rapport à la célèbre somme d'Izikowitz (1935). Ce tableau d'ensemble des instruments de l'Amérique centrale et du Sud fut, on le sait, établi à partir de collections de musées européens. Si les travaux actuels utilisent toujours ce livre comme source documentaire fondamentale, certaines assertions appellent des corrections ou des compléments. Par exemple, les données récentes attestent la nature précolombienne, amazonienne même, des grandes clarinettes alors qu'Izikowitz, disposant de peu de références, leur attribuait une origine européenne (Beaudet 1989).

Mais surtout on commence, à la fin des années 70 , à se dégager d'une simple liste d'instruments, d'un simple inventaire des éléments musicaux ${ }^{1}$, pour tenter d'intégrer ceux-ci dans une production musicale, dans un discours sur la musique, enfin dans un monde sonore considéré lui-même comme signifiant.

Menezes Bastos (1978) donne une intéressante approche des conceptualisations musicales kamayura centrées sur des notions comme celle de " courant sonore », 
et dont le vocabulaire semble particulièrement riche en regard des autres ethnies amazoniennes. La présentation taxinomique qu'en propose cet auteur est, pour l'Amérique du Sud amérindienne, la première tentative d'analyse d'une pensée musicale.

Seeger (1981, 1987), en s'appuyant comme André Schaeffner sur des questions « maussiennes » (quoi, qui, où, quand, comment ?) opère une avancée épistémologique significative pour l'ensemble de la discipline. S'il nous laisse sur notre faim quant aux nécessaires caractérisations musicales, c'est que son projet est d'analyser la musique d'abord comme une production plutôt que comme un produit. Parmi les différents thèmes abordés par Seeger, un des plus remarquables est celui qui fait apparaître la musique comme un moyen de définition et d'expression de l'identité sociale des individus et des groupes cérémoniels : chez les Suyá, l'initiation des garçons, la résidence uxorilocale et la vie cérémonielle font que, pour un homme, ses « sœurs » sont à la fois ses parentes privilégiées et les personnes auxquelles il ne peut pas communiquer directement ses états émotionnels. Il n'y parvient que grâce à un type de chant - akia - dont les caractéristiques musicales favorisent la différenciation individuelle; c'est un chant de groupe, mais en hétérophonie, où simultanément chaque homme énonce son propre répertoire d'une voix aiguë, tendue, tandis que les femmes - «les sœurs »- écoutent, reconnaissent et commentent. Par contraste, les ngere, chants collectifs des groupes cérémoniels, réaffirment la valeur de l'unité communautaire grâce à des caractéristiques musicales opposées à celles du chant akia : recherche de l'unisson, homogénéité des timbres des voix...

Comme le souligne Seeger, dans des organisations sonores de ce type, le silence - ne pas prendre part à une activité musicale - est en soi hautement signifiant ; on exprimera ainsi une tristesse personnelle ou, plus souvent, une prise de position politique. Cette conception de l'acte musical comme signe circonstanciel plutôt que comme catégorie fonctionnelle, sans être une singularité de l'Amazonie, semble y être particulièrement fréquente et active.

Trois travaux plus récents - une thèse et deux disques - permettront de présenter ici quelques thèmes qui sont au cœur de l'ethnomusicologie amazoniste contemporaine : la question de la sémanticité de la musique, la relation entre centre et périphérie, musique et histoire...

La problématique de la sémantique musicale est abordée de front par Menezes Bastos dans sa thèse, $A$ Festa da Jaguatirica : uma partitura crítico-interpretativa (1989), qui est une interprétation du yawari, rituel de deuil d'origine trumai ${ }^{2}$, mis en œuvre ici par les Kamayura du Parc national du Xingu au Brésil. La jalousie, qui en constitue un des thèmes principaux, est mise en scène au moyen d'oppositions multiples qui, selon l'auteur, sont contredites dans la musique par des jeux de « miroirs mélodiques » et de diversifications tonales. Le yawari, comme les autres rituels du haut Xingu, est considéré comme une lingua franca interethnique (Menezes Bastos 1978) qui canalise l'agressivité intertribale (Galvão 1979), mais aussi qui « met en scène la mort comme double de l'échange cosmico-social » (Menezes Bastos 1989 : 347). Basée sur une transcription de la musique et des paroles, cette étude se focalise sur les éléments tonaux et les motifs (mélodiques), sans prendre en compte les rythmes, les danses et les parures corporelles.

La question de la sémanticité de la musique, centrale dans ce travail, est traitée d'abord d'un point de vue théorique : c'est l'histoire socio-religieuse des musicologies 
qui a " expulsé le signifié de la musique pour la condamner au simple contexte " (p. 46) (par opposition au verbe biblique qui a le monopole du sens, la musique se sent mais ne s'explique pas).

Dans l'interprétation de l'ethnographie du yawari, Menezes Bastos prend le parti d'une analyse musicologique la plus fine possible afin de sortir d'une prétendue monotonie de ces musiques et d'y trouver le signifié. Pour lui, échelles et cadres scalaires, motifs et cadres mélodiques sont ici signifiants (par eux-mêmes et dans leurs interrelations). Cette « sémiologie mélodico-tonale » met en évidence un ensemble d'oppositions structurées sur un intervalle mélodique ou sur une relation entre un intervalle et le centre tonal. Ainsi, une chanson de la séquence d'ouverture de ce rituel oppose deux conceptions de la mort correspondant aux deux grands rituels de deuil, le $k w a r y p$ et le yawari, par une ambivalence tonale et mélodique « toujours, pourtant, résolue dans l'unicité, ou plutôt dans la prépondérance du centre tonal et des dessins affirmés sous son poids " (p. 275). Autre exemple pris dans la séquence de l'Aurore « les motifs de cette incroyable Aurore - où la 'joie' s'oppose à la 'tristesse' associée à la 'colère', mais aussi emboutit la 'peur' et la 'douleur' - sont pour ainsi dire libérés d'une prison tono-modale univoque. Il n'y a plus d'axes de centres tonaux en constitution ou en affirmation, mais les axes 'motiviques' de l'Aurore supposent des motifs parfois très différents sur le plan tonal » (p. 384). Associer ainsi une « libération tonale " à l'affirmation de la joie est une interprétation brillante, mais qu'il nous est parfois difficile, comme pour d'autres interprétations de cette thèse, de mettre en relation avec les données de terrain. Si l'auteur ne propose pas de synthèse de cette " polémique tonale », il semble bien qu'elle soit prépondérante dans l'ensemble des chants et qu'elle renvoie au jeu complexe des oppositions mises en scène par le rituel : amphytrions/invités, parallèles/croisés, Indien/non-Indien, amant/mari jaloux, sexe plaisir/sexe reproductif, humains/divins, mort valeureuse/mort laide... Toutefois, dans la plupart des chants, la musique « contredit » ces oppositions strictes, " critique » ce côté manichéen exprimé par les paroles, en utilisant par exemple trois échelles et non deux, comme dans l'ouverture, " multipliant ainsi les plans de similitude et de différence entre rite et récit ».

On peut regretter que l'auteur considère les tensions entre les deux principaux protagonistes du rituel comme une perturbation de celui-ci au lieu de les analyser comme conflit significatif, mais il interprète de manière très intéressante les attitudes des différents acteurs où il voit une volonté « d'intervenir dans le temps mythique " pour faire de chaque « fête du yawari un commencement antérieur à celui du mythe ». Celui-ci, raconté par un des acteurs pendant le rituel, dit que l'amant, créateur de jalousie, déshonoré devant ses maîtresses par le mari, s'exile longtemps et va au loin " chercher la jalousie et l'envie, non plus comme choses en soi, mais comme objets rituels : c'est le yawari » (p. 572). « Pour les Kamayura, les paroles 'entrent' dans la musique qui elle-même 'entre' dans le corps dansant. Cette 'entrée' ressemble à une traduction. Du mythe à la danse, c'est un seul univers de signifiés liés à trois mondes de signifiants équivalents " (p. 523). Dans l'anthropologie d'aujourd'hui, il me semble encore nécessaire d'appuyer cette thèse : la musique est un système de représentations à part entière, lui-même relié aux autres systèmes, par exemple le religieux ou l'organisation sociale, sans qu'il soit possible d'établir des relations successives entre les uns et les autres. 
La relation entre musique et société semble une fois de plus inséparable de la question du sens musical et renvoie ainsi à des thèmes fondamentaux tels celui de la forme des discours, en elle-même porteuse de significations (Basso 1985 ; Sherzer \& Urban 1986). Dans un même groupe, les différentes organisations sonores - de la conversation quotidienne au chant d'initiation, par exemple - forment système ; et ce, en association avec l'organisation de l'espace. Ces agencements mettent en jeu les conceptions du centre et de la périphérie, du social et de l'asocial, de l'humain et du non-humain... en exploitant de manière variable et distinctive les différentes ressources de la langue et de la musique. Seeger (1987 : 25-51, 115-118) montre comment les Suyá mettent en scène ces équivalences dans leur cérémonie de la souris. Graham (1986) analyse trois formes d'expression vocale chez les Xavante - les plaintes rituelles, les chants collectifs et les discours politiques - et y fait apparaître une différenciation à la fois linguistique, musicale et socio-spatiale : alors que les plaintes n'utilisent que trois phonèmes, les chants collectifs exploitent tout l'éventail morphophonologique, mais sans contenu sémantique, tandis que les discours ont, bien sûr, un contenu sémantique ; des plaintes aux discours, les possibilités musicales (hauteurs, complexité mélodique) sont de moins en moins exploitées ; enfin, dans chaque cas, les lieux et les acteurs contribuent à définir un continuum de la périphérie (l'espace domestique) au centre (l'espace social). Dans le rituel du yawari des Kamayura, cette systématisation de la relation centre/périphérie est prise en charge par « une symphonie d'onomatopées de probablement tous les animaux emblématiques du yawari » (Menezes Bastos 1989 : 112, 351). Ici, l'environnement naturel acquiert, grâce à ces onomatopées ${ }^{3}$, une présence sonore à la fois périphérique et équivalente à celle du noyau même de la scène rituelle.

Le disque Brésil central : chants et danses des Indiens Kaiapó donne des exemples sonores d'une opposition « social-asocial » qui pourrait s'inscrire dans un continuum village-forêt et qui se traduit par un jeu d'oppositions - centre du village, périphérie, forêt, forêt éloignée. Ce jeu est exprimé par la musique qui peut tantôt amener « l'asocial » au centre du village, tantôt socialiser la vie en forêt : ainsi de la cueillette des lianes (AIMP XIV : 10) où chants, bruits de la cueillette et sons de la forêt se mêlent magnifiquement. Des enregistrements de ce type sont essentiels pour comprendre les musiques d'Amazonie.

La musique kaiapo est principalement vocale. Ces chants, comme quelques autres sur ces disques, furent enregistrés lors de répétitions quotidiennes. Précision importante dans la mesure où la présence ou l'absence d'un apprentissage formalisé, critère peu analysé pour caractériser les musiques des basses terres, peut aider à dégager des typologies socio-musicales indigènes. Il est fréquent que, selon la circonstance - apprentissage ou cérémonie -, le même chant ne soit pas exécuté selon les mêmes paramètres musicaux : ainsi, chez les Kaiapo, les chants le sont dans le registre grave pendant les répétitions et dans un registre très aigu lors de la cérémonie elle-même.

On peut dire que ces chants kaiapo correspondent à l'esthétique musicale prépondérante chez les Gé : homophonie, prédominance de l'émission recto tono (ou sur très peu de hauteurs) avec transpositions fréquentes, valeurs rythmiques égales sur un tempo rapide avec accentuation très marquée, le résultat étant un débit haché et puissant, typique des chants collectifs gé ( $c f$. disque Suyá, et Graham 1986). Cette 
tentative de généralisation doit, bien sûr, être relativisée, et ce d'abord à l'intérieur de chaque groupe ; ainsi le chant des femmes xikrin (AIMP XV :2) présente, selon les séquences, des différences significatives du mode d'accentuation.

Autre recueil de musiques gé, Le Monde sonore des Bororo est un disque étonnant par ses qualités émotionnelles et esthétiques ainsi que par son originalité. Exhortations, oraisons récitées, cris, chants en solo ou en chœur... la voix est, comme pour les Kaiapo et probablement tous les Gé, à l'avant-scène de l'art sonore bororo. Les instruments de musique offrent, selon l'auteur du disque, «peu de possibilités de variations de timbres ou de hauteurs ». Toutefois les hochets, presque omniprésents, ont une fonction structurante pour les chants qu'ils accompagnent. L'analyse des rythmes joués avec ces hochets semble nécessiter une connaissance pratique de leur technique complexe de jeu. Ces chants bororo apparaissent, dans leur ensemble, différents des autres " musiques gé »; seuls les premiers chants de l'oieigo (plage 9) sont comparables aux chants des autres Gé par leur rythme et, plus précisément, par leur mode d'accentuation (Canzio 1992).

Dernier thème abordé : la relation entre musique et histoire. L'ethnomusicologie amazoniste est loin de pouvoir proposer une musico-chronologie exploitable par des ethnohistoriens, même si Menezes Bastos fait une modeste tentative dans cette direction (1989: $529 s q$.). En ce domaine, on ne peut encore établir que des correspondances ponctuelles (Seeger 1990). Ainsi, le coffret de disques kaiapo est consacré aux Mekrãgnoti et aux Xikrin. Ces deux communautés, ayant fait scission au début du Xvirle siècle, " passent pour être les plus éloignées l'une de l'autre du point de vue géographique, historique et même culturel. Mais, en dépit de cette distance, les expressions musicales des Mekrãgnoti et des Xikrin sont très proches » (ibid.). Par ailleurs, les différents enregistrements de ce coffret permettent des comparaisons diachroniques entre ceux des années 1978-1980, ceux de 1966 et ceux effectués par S. Dreyfus en 1955 (voir discographie). On constatera peut-être la disparition ou la simplification de certains éléments du répertoire, mais, en contrepartie, la vigueur démographique de ces groupes est sensible dans l'exécution actuelle de ces chants et danses. Ces deux caractéristiques conjointes - perte apparente et vigueur de l'interprétation -, que l'on retrouve chez d'autres peuples amérindiens, peuvent constituer des paramètres fructueux pour l'analyse des contacts entre les groupes indigènes et les fronts de colonisation.

Les Bororo, eux, ont à gérer ce contact depuis près de trois siècles. Leur culture matérielle emprunte beaucoup à celle de la civilisation moderne environnante, mais dans leur musique rituelle telle qu'elle est présentée sur le disque qui leur est consacré, on ne peut déceler de transformation qui serait liée à la colonisation. Ces extraits semblent donc confirmer la forte étanchéité des systèmes sonores indigènes des basses terres d'Amérique du Sud par rapport aux musiques non amérindiennes, citadines ou paysannes. Mais surtout, par sa richesse sonore, ce disque nous plonge dans la densité rituelle, contrepoint d'un vide quotidien très actuel, produit d'abord par les dramatiques conditions de contact ${ }^{4}$. La musique peut-elle nous aider à distinguer dans ce contraste violent la part de l'histoire contemporaine et celle d'une esthétique, peut-être "très gé », de l'irruption et du vide ?

Ainsi, malgré son retard, l'ethnomusicologie amazoniste commence-t-elle à proposer des lignes de réflexion nouvelles. S'il reste nécessaire et urgent de développer 
la collecte et l'ethnographie de ces musiques ${ }^{5}$, celles-ci, pour être comprises, exigent qu'on dépasse le stade des analyses formelles et qu'on intègre matériaux et pratiques sonores dans l'ensemble des représentations sociales et cosmologiques.

Université de Paris $X$-Nanterre

\section{NOTES}

1. Voir les monographies descriptives de Halmos (1979) et Aytai (1985).

2. On peut entendre une splendide interprétation d'un chant du yawari par les Trumai dans le disque Brésil : musique du haut Xingu.

3. Par ailleurs, comment mettre en rapport la sémanticité de la musique et celle de la langue sans évaluer la proportion, la place, les types de référents, des onomatopées dans le lexique et dans les textes d'une langue donnée ? Ainsi, kyry, une petite séquence chantée de ce rituel, est une onomatopée des odeurs sexuelles : "icône sonore au signifié olfactif "! (p. 158).

4. Voir par exemple le film Autour de Tristes tropiques réalisé par Patrick Menget et Jean-Pierre Beaureneaut en 1991.

5. Aujourd'hui ces études s'amplifient - multiplication des mémoires universtaires, en particulier au Brésil -, mais nous ne savons pratiquement rien, par exemple, des musiques pano ou arawak.

\section{RÉFÉRENCES}

AYTAI, Desiderio

1985 O Mundo sonoro Xavante. São Paulo, Universidade de São Paulo.

Basso, Ellen B.

1985 A Musical View of the Universe : Kalapalo Myths and Ritual Performance. Philadelphia, University of Pennsylvania Press.

BEAUDET, Jean-Michel

1989 «Les turè, des clarinettes amazoniennes » Revista de Música latino americana 10/1 : 92-115. Austin.

CAMEU, Helza

1977 Introdução ao estudo da música indígena brasileira. Rio de Janeiro, Conselho Federal de Cultura.

CANZIO, Ricardo

1992 "Mode de fonctionnement rituel et production musicale chez les Bororo du Mato Grosso », Cahiers de Musiques traditionnelles 5 : 71-96.

GaLVĀo, Eduardo

1979 Encontro de sociedades. Rio de Janeiro, Paz e Terra.

GRAHAM, Laura

1986 «Three Modes of Shavante Vocal Expression : Wailing, Collective Singing, and Political Oratory ", in J. SHERZER \& G. URBAN, eds., Native South American Discourse : 83-118.

HaLmos, Istvan

1979 The Music of the Nambicuara Indians (Mato Grosso, Brazil). Budapest, Akademiai Kiado (« Acta Ethnographica Academiae Scientarum Hungaricae» 28 (1-4)) : 205-350. 
D'Harcourt, Raoul \& Marguerite

1925 La Musique des Incas et ses survivances. Paris, Geuthner.

1959 La Musique des Aimara sur les hauts plateaux boliviens. Paris, Société des Américanistes.

IzIKowITZ, Karl Gustav

1935 Musical and Other Sound Instruments of the South American Indians. Göteborg, Elanders Boktryckeri.

MENEzes Bastos, Rafael José DE

1978 A Musicológica Kamayura : para uma antropologia da communicaçáo no Alto Xingu. Brasília, Funai.

1989 A Festa da Jaguatirica : uma partitura crítico interpretativa. São Paulo, Thèse de Doctorat, ms.

SEEGER, Anthony

1979 "What Can we Learn when they Sing ? Vocal Genres of the Suyà Indians of Central Brazil », Ethnomusicology 23 : 373-394.

1987 Why Suyá Sing : A Musical Anthropology of an Amazonian People. Cambridge, Cambridge University Press.

1991 «When Music Makes History », in S. Blum, P. Bohlman \& D. NeumaN, eds., Ethnomusicology and Modern Music History. Urbana \& Chicago, University of Illinois Press : 23-35.

SHERZER, J. \& G. URBAN, eds.

1986 Native South American Discourse. Berlin, Mouton.

\section{DISCOGRAPHIE CITÉE}

Brésil : le monde sonore des Bororo. Un CD : enregistrements et texte : Ricardo Canzio ; photographies : Priscilla Ellis. AUVIDIS-UNESCO D 8201 (1989). Durée totale : $78 \mathrm{mn} 27 \mathrm{~s}$.

Brésil central : chants et danses des Indiens Kaiapó. Coffret de deux CD ; enregistrements : René Fuerst et Georges Love (1966), Pascal Rosseels (1974-1975), Gustaaf Verswijver (1978-1981) ; texte : G. Verswijver ; photographies : R. Fuerst, G. Verswijver. AIMP XIV-XV, CD 554-555 (1989). Durée totale : $120 \mathrm{mn}$.

Brésil : musique indienne. Un disque $33 \mathrm{t}, 30 \mathrm{~cm}$; enregistrements et notice : Simone Dreyfus. Musée de l'Homme - Vogue LD-15 (1957), rééd. 1969 et 1972, Paris.

A Arte Vocal dos Suyá. Un disque $33 \mathrm{t}, 30 \mathrm{~cm}$; enregistrements : J. et A. Seeger ; notice : Anthony Seeger. Tacape T007 (1982), São João Del Rei.

Brésil : musique du Haut Xingu. Enregistrements : J.-F. Schiano ; notice : Patrick Menget. Ocora C 580022 (1992), Paris. 